\title{
Lateral Femoral Circumflex Arterial System as Donor Vessels for Extremity Replantation
}

\author{
Erin M. Taylor, MD ${ }^{1} \quad$ Matthew L. Iorio, MD ${ }^{1}$ \\ ${ }^{1}$ Division of Plastic Surgery, Beth Israel Deaconess Medical Center, \\ Department of Surgery, Harvard Medical School, Boston, Massachusetts \\ Address for correspondence Matthew L. Iorio, MD, Division of Plastic \\ Surgery and Department of Orthopaedic Surgery, Beth Israel Deaconess \\ Medical Center, Harvard Medical School, 330 Brookline Avenue, Stoneman \\ J Reconstr Microsurg Open 2016;1:88-91. \\ 10, Boston, MA 02215 (e-mail: matt.iorio@bidmc.harvard.edu).
}

\begin{abstract}
\section{Keywords}

- extremity replantation

- LFCA

- vascular grafting

Background Forearm replantation presents with many challenges, including the need for vascular graft material for revascularization. Although frequently harvested for vascular reconstruction, the saphenous vein is often suboptimal for use as vascular conduit due to peripheral vascular disease, atherosclerosis, or size and length limitations.

Methods We describe the use of the lateral femoral circumflex arterial (LFCA) system for arterial and venous graft in the case of a proximal forearm replantation.

Results The LFCA system provided significant amounts of arterial and venous graft after the saphenous vein was found to be suboptimal for conduit in the case of forearm replantation. Postoperatively, the patient developed wound-healing complications at the saphenous vein harvest site but not at the LFCA donor site. The forearm revascularization was successful with recovery of sensation in his digits by 15 months postoperative.

Conclusion The LFCA system is a viable and possible superior alternative to the saphenous vein in vascular reconstruction, providing significant amounts of venous and arterial graft with potentially decreased donor site morbidity and through a well-known approach.
\end{abstract}

Forearm replantation presents with many challenges including the requirement of significant amounts of vascular graft for revascularization. The saphenous vein is frequently harvested for use as a conduit in vascular reconstruction. However, many patients have suboptimal saphenous veins due to peripheral vascular disease and atherosclerosis, which places the vascular reconstruction and thus whole replant or flap survival, at risk.

Less frequently, the lateral femoral circumflex arterial (LFCA) system is described for use in vascular reconstruction. Prior use of LFCA grafts in the literature include interposition grafts for head and neck free flaps, ${ }^{1,2}$ lower extremity reconstruction, ${ }^{3}$ added pedicle length to prefabricated flaps, ${ }^{4}$ alternative to arteriovenous loops for flaps distant to recipient vessels, ${ }^{4}$ ulnar artery reconstruction in hypothenar hammer syndrome, ${ }^{5,6}$ and extremity flow-through revascularization. ${ }^{4}$

received

May 26, 2016

accepted

June 7, 2016

published online

August 17, 2016
We describe the use of the LFCA system for multiple arteriovenous grafts in the case of a proximal forearm replantation.

\section{Case}

The patient was a 74-year-old right-hand dominant man who presented with a near-complete proximal-third forearm amputation from entrapment in a log splitter. At the time of the injury, the patient placed a tourniquet. The emergency medical services team briefly let down the tourniquet on arrival to evaluate the injury; however, they immediately reapplied it due to considerable blood loss. On arrival to the hospital, the patient denied known medical problems and was emergently taken to the operating room for attempted forearm replantation.
Copyright $\odot 2016$ by Thieme Medical Publishers, Inc., 333 Seventh Avenue, New York, NY 10001, USA. Tel: +1(212) 584-4662.
License terms $10.1055 / \mathrm{s}-0036-1587338$ ISSN $2377-0813$.

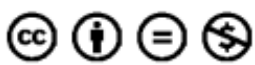




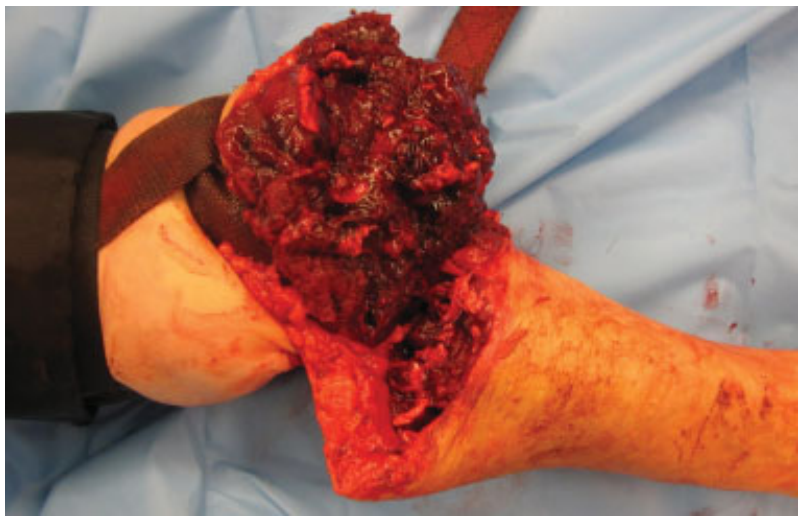

Fig. 1 Preoperative forearm with a near-complete proximal-third forearm amputation from entrapment in a log splitter and tourniquet in place. A skin bridge, approximately $1.5 \mathrm{~cm}$ long, was present without intervening arterial, venous, or neural supply.

Intraoperatively, evaluation of the left upper extremity revealed a thin, tenuous skin bridge, approximately $1.5 \mathrm{~cm}$ long, without intervening arterial, venous, or neural supply (-Fig. 1). The skin bridge was transected and a two-team approach to forearm replantation was initiated. The extremity part underwent debridement of nonviable, crushed, or contaminated muscle and skin on the back table. A longitudinal incision was made just ulnar to the radial artery neurovascular bundle, which was proximally traced. The radial artery and veins were dissected and thrombosis transected. The radial sensory branch was identified and distally prepared. Similarly, the median nerve and the ulnar artery, vein, and nerve were prepared with resection of thrombus or nonviable tissue until healthy appearing tissue was encountered. The radius and ulna, which were fractured transversely with some comminution, were shortened by approximately $4 \mathrm{~cm}$.

With attention turned to the left forearm amputation stump, all nonviable muscle and skin were debrided. The brachial artery in the proximal stump was divided into a radial artery and an ulnar artery, which were both identified and dissected. The radial, median, and ulnar nerves were identified, tagged, and trimmed back to healthy appearing tissue. The ulnar artery, which was the largest artery on the amputated part, was cannulated with a 6-French tube and connected to the ulnar artery of the stump. The tourniquet was released to reestablish flow for a total ischemia time since injury of 3.5 hours. Osteosynthesis was completed with a $3.5-\mathrm{mm}$ locking compression plate on both the radius and ulna. Although it appeared that nerve repairs would be coapted without tension, the zone of injury of the vascular repairs would require a venous graft.

Attention was turned to the lower extremity for vein graft where a longitudinal incision was made in the medial lower leg, and Doppler ultrasound was used to confirm subcutaneous veins; however, the patient had significant varicosities and peripheral vascular disease. The saphenous vein appeared heavily sclerosed and unusable for vascular reconstruction. Attention was instead turned to the thigh to examine the LFCA system for donor vessels. A longitudinal line was made from the anterior superior iliac spine to the superolateral patella, and the plane

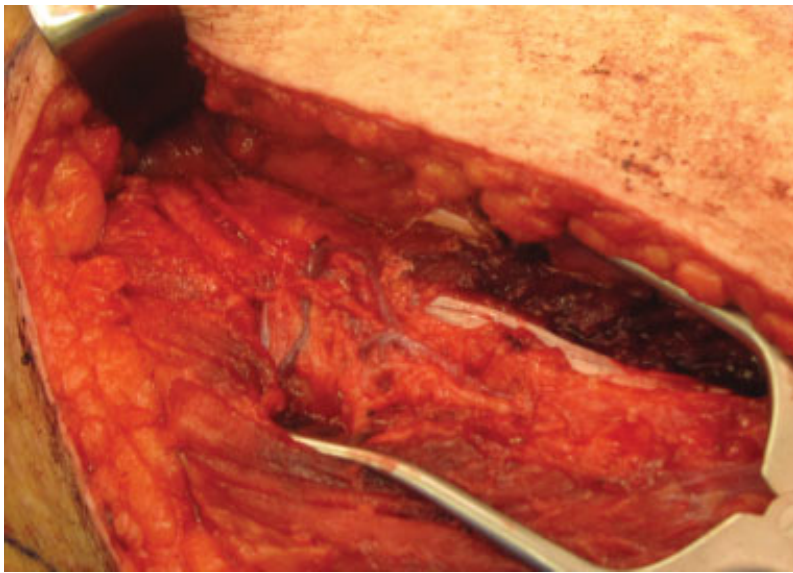

Fig. 2 Lateral femoral circumflex arterial (LFCA) system as donor artery and veins. The LFCA system was circumferentially dissected from distal to proximal of the venae comitantes, artery, and transverse branch, which were harvested for interposition grafts in forearm revascularization.

between the rectus femoris and vastus lateralis was dissected until the descending branch of the LFCA system was identified. The LFCA system was circumferentially dissected from distal to proximal of the venae comitantes, artery, and transverse branch, which were harvested as vein and arterial grafts (-Fig. 2).

Vascular reconstruction first focused on the radial artery of the stump and part, using a 4-cm reverse vein graft from the LFCA system with 9-0 nylon sutures. The radial comitantes were anastomosed in similar fashion using 4-cm grafts from the venae comitantes of the LFCA system with 9-0 nylon sutures. A venous repair with another 4-cm vein graft was performed with the anterior interosseous neurovascular bundle, which had a high volume of venous back bleeding. After pulsatile radial flow was confirmed with Doppler, the ulnar artery shunt was clamped proximally and removed distally. Residual clot that had surrounded the shunt was removed with no. 2 Fogarty catheter. A 4-cm LFCA graft was utilized to repair the ulnar artery with 9-0 nylon sutures. The cephalic vein was identified and anastomosed with a 4-cm vein graft and couplers and lastly, another volar vein was identified and coupled with a $4-\mathrm{cm}$ vein graft (-Fig. 3). The radial sensory nerve, median nerve and ulnar nerve were repaired with tension-free coaptations. The skin was loosely closed and a dressing was applied.

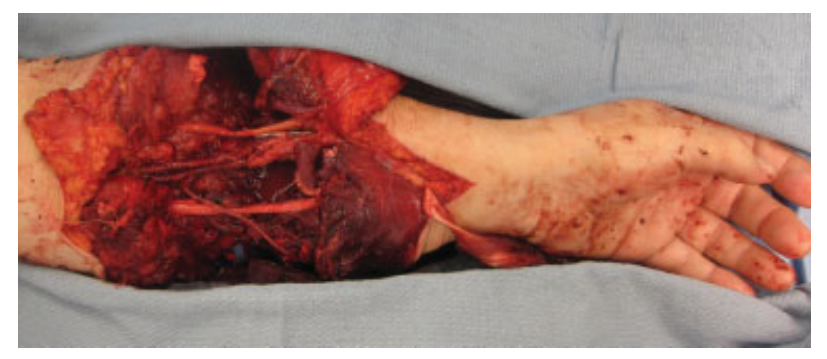

Fig. 3 Forearm replant after osteosynthesis, nerve repair, arterial and venous repair with interposition grafts from the lateral femoral circumflex arterial system, and reestablishment of blood flow with tourniquet release. Total ischemia time from injury was 3.5 hours. 
A

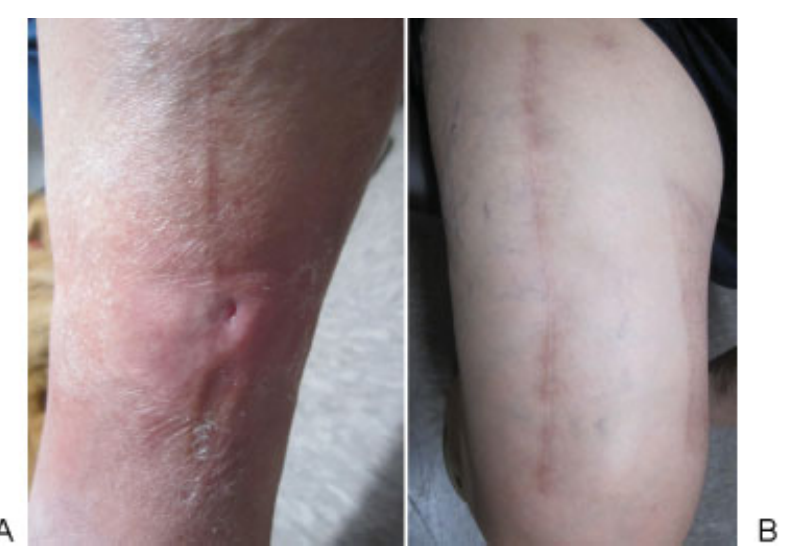

Fig. 4 Postoperative sites of the (A) saphenous and (B) lateral femoral circumflex arterial (LFCA). The saphenous donor site had wound breakdown whereas the LFCA donor site healed without complication.

The patient recovered well from the forearm replantation, maintaining good distal forearm arterial inflow and venous outflow. The patient returned to the operating room 3 days later for an Achilles allograft reconstruction of the volar forearm muscle belly of the flexor digitorum profundus (FDP) to a side-to-side tenodesis of the proximal FDP tendon stumps. The remaining open areas were closed with split-thickness skin grafts. Subsequent surgeries included revision osteosynthesis of the radius and ulna, and ulnar shaft revision osteosynthesis with iliac crest bone graft. He had wound-healing difficulties with the saphenous vein harvest site but not with the LFCA donor site ( $\mathbf{F i g . 4 A , ~ B ) . ~ A t ~} 15$ months postoperatively, the patient had recovery of sensation in his volar and dorsal digits, palm, and distal and proximal forearm ( - Fig. 5).

\section{Discussion}

Forearm replantation involves the complex reconstruction of many structures in the upper extremity. Vascular reconstruction often involves autograft conduits to ensure that the anastomosis lacks tension. The saphenous vein is the standard conduit harvested but can be compromised due to peripheral vascular disease, as seen with our case patient, which may compromise revascularization. Conversely, the descending branch of the LFCA system is often spared from peripheral vascular disease. In a retrospective review examining angiograms of patients with suspected peripheral vascular disease, Halvorson et al found that the descending branch of the LFCA system was spared from atherosclerosis in $87 \%$ of patients. ${ }^{7}$

The LFCA system is located along a longitudinal line connecting the anterior superior iliac spine to the superolateral patella. The descending branch of the LFCA system can be found within the plane between the rectus femoris and vastus lateralis. In a cadaveric study by Zenn et al, the descending branch of the LFCA system typically has a mean pedicle length of $20.5 \mathrm{~cm}$, proximal arterial diameter of $3.4 \mathrm{~mm}$, distal arterial diameter of $1.9 \mathrm{~mm}$, and proximal venous diameter of $3.9 \mathrm{~mm}$. Of the eight fresh legs of the five cadavers studied, $60 \%$ percent of had two sizable venae comitantes, ${ }^{4}$ providing vessel of ample size and length for reconstruction.

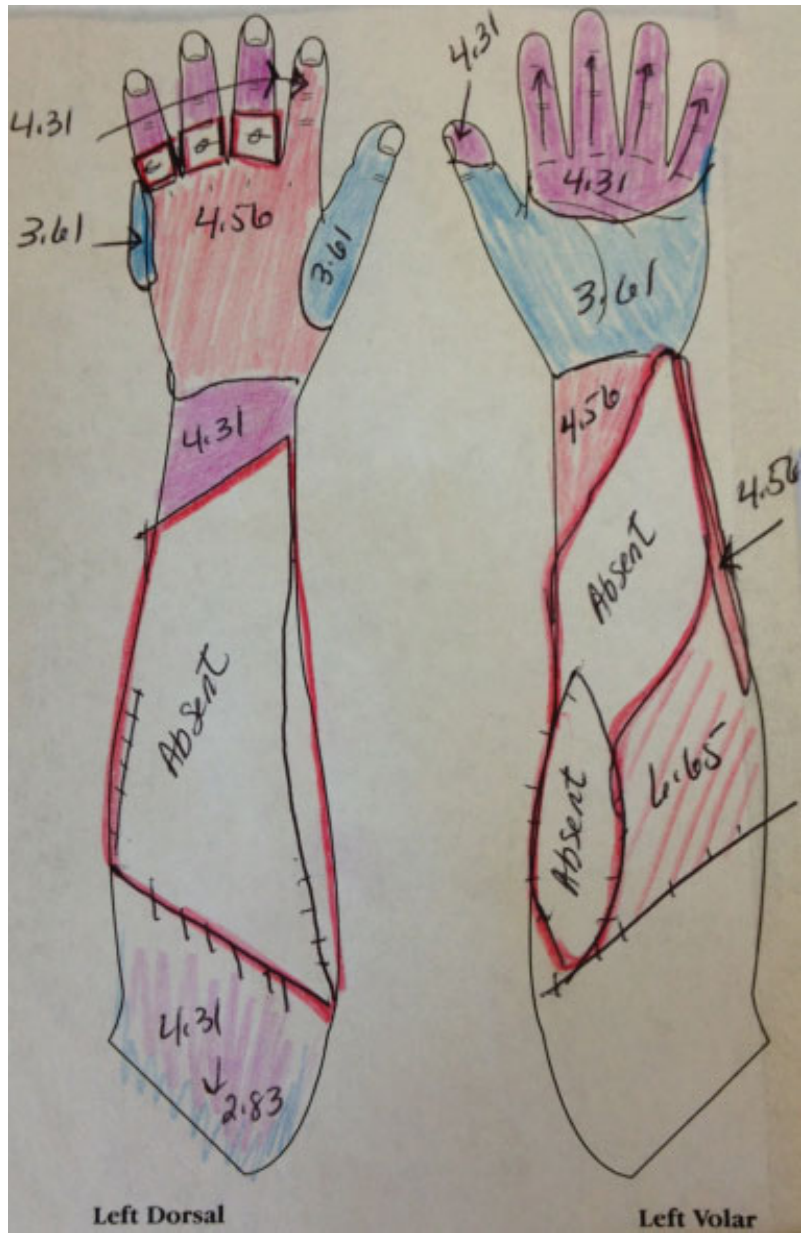

Fig. 5 Sensory recovery 15 months postoperative. Evaluator sizes listed on image of $2.83,3.61,4.31,4.56$, and 6.45 correspond to target forces of $0.07,0.4,2.0,4.0$, and $180 \mathrm{~g}$, respectively. Colors correspond to threshold of sensation: blue, diminished light touch; purple, diminished protective sensation; red, loss of protective sensation; red lined, tested with no response.

Furthermore, the LFCA system provides both arterial graft in addition to the venae comitantes grafts, which give the LFCA system a significant advantage over venous grafts in arterial reconstruction. Arterial conduit grafts have significantly increased patency rates compared with venous conduits in distal upper extremity bypass surgery. ${ }^{8}$ Masden et al examined 152 grafts in upper extremity revascularization (19 of 152 were arterial conduits) in a systematic literature review, finding rates of $100 \%$ arterial conduit patency and $85 \%$ vein graft patency, with a statistically significant difference in patency rates. $^{8}$

The LFCA system provided an advantageous alternative to the saphenous vein graft, contributing significant amounts of vascular conduit, including arterial graft that may improve chances of replantation patency. The limitation of the LFCA system as a donor site is its inability to use the the fasciocutaneous anterolateral thigh (ALT) flap of the ipsilateral leg; however, the contralateral leg can provide an ALT flap for reconstruction if needed. 


\section{Conclusion}

The LFCA system is a viable and possible superior alternative to the saphenous vein in vascular graft reconstruction, providing significant amounts of venous and arterial graft with potentially decreased donor site morbidity.

\section{References}

1 Park JH, Min KH, Eun SC, Lee JH, Hong SH, Kim CW. Scalp free flap reconstruction using anterolateral thigh flap pedicle for interposition artery and vein grafts. Arch Plast Surg 2012;39(1):55-58

2 Bianchi B, Copelli C, Ferrari S, Ferri A, Sesenna E. Anterolateral thigh flap pedicle for interposition artery and vein grafts in head and neck reconstruction: a case report. Microsurgery 2009;29(2):136-137
3 Echo A, Bullocks JM. Use of the descending branch of the lateral femoral circumflex vessels as a composite interposition graft in lower extremity reconstruction. Microsurgery 2011;31(3):241-245

4 Zenn MR, Pribaz J, Walsh M. Use of the descending lateral femoral circumflex artery and vein for vascular grafting: a better alternative to an arteriovenous loop. Plast Reconstr Surg 2010;126(1):140-142

5 de Niet A, Van Uchelen JH. Hypothenar hammer syndrome: long-term follow-up after ulnar artery reconstruction with the lateral circumflex femoral artery. J Hand Surg Eur Vol 2015 [Epub ahead of print]

6 Dethmers RS, Houpt P. Surgical management of hypothenar and thenar hammer syndromes: a retrospective study of 31 instances in 28 patients. J Hand Surg Br 2005;30(4):419-423

7 Halvorson EG, Taylor HO, Orgill DP. Patency of the descending branch of the lateral circumflex femoral artery in patients with vascular disease. Plast Reconstr Surg 2008;121(1):121-129

8 Masden DL, Seruya M, Higgins JP. A systematic review of the outcomes of distal upper extremity bypass surgery with arterial and venous conduits. J Hand Surg Am 2012;37(11):2362-2367 